\title{
A method for high precision sequencing of near full-length 16S rRNA genes on an Illumina MiSeq
}

\author{
Catherine M Burke Corresp., $^{1}$, Aaron E Darling ${ }^{1}$ \\ ${ }^{1}$ The i3 institute, University of Technology Sydney, Sydney, NSW, Australia \\ Corresponding Author: Catherine M Burke \\ Email address: Catherine.Burke@uts.edu.au
}

Background. The bacterial 16S rRNA gene has historically been used in defining bacterial taxonomy and phylogeny. However, there are currently no high-throughput methods to sequence full-length 16S rRNA genes present in a sample with precision.

Results. We describe a method for sequencing near full-length 16S rRNA gene amplicons using the high throughput Illumina MiSeq platform and test it using DNA from human skin swab samples. Proof of principle of the approach is demonstrated, with the generation of 1604 sequences greater than $1300 \mathrm{nt}$ from a single Nano MiSeq run, with accuracy estimated to be 100 -fold higher than standard Illumina reads. The reads were chimera filtered using information from a single molecule dual tagging scheme that boosts the signal available for chimera detection.

Conclusions. This method could be scaled up to generate many thousands of sequences per MiSeq run and could be applied to other sequencing platforms. This has great potential for populating databases with high quality, near full-length 16S rRNA gene sequences from under-represented taxa and environments and facilitates analyses of microbial communities at higher resolution. 


\section{A method for high precision sequencing of near full-length 16S rRNA genes on an Illumina}

\section{MiSeq}

3 Catherine M. Burke ${ }^{1 *}$ and Aaron E. Darling ${ }^{1 *}$

4 1. The ithree institute, University of Technology Sydney, NSW, Australia

5

$6 *$ Corresponding author: Catherine Burke, email: catherine.burke@uts.edu.au

7 * Corresponding author: Aaron Darling, email: aaron.darling@uts.edu.au 8

9 


\section{ABSTRACT}

11 Background. The bacterial 16S rRNA gene has historically been used in defining bacterial 12 taxonomy and phylogeny. However, there are currently no high-throughput methods to sequence 13 full-length 16S rRNA genes present in a sample with precision.

14 Results. We describe a method for sequencing near full-length 16S rRNA gene amplicons using 15 the high throughput Illumina MiSeq platform and test it using DNA from human skin swab 16 samples. Proof of principle of the approach is demonstrated, with the generation of 1604 17 sequences greater than $1300 \mathrm{nt}$ from a single Nano MiSeq run, with accuracy estimated to be 18 100-fold higher than standard Illumina reads. The reads were chimera filtered using information 19 from a single molecule dual tagging scheme that boosts the signal available for chimera 20 detection.

21 Conclusions. This method could be scaled up to generate many thousands of sequences per 22 MiSeq run and could be applied to other sequencing platforms. This has great potential for 23 populating databases with high quality, near full-length 16S rRNA gene sequences from under24 represented taxa and environments and facilitates analyses of microbial communities at higher 25 resolution.

\section{INTRODUCTION}

Amplifying and sequencing 16S rRNA genes from microbial communities has become a standard technique to survey and compare communities across space, time and environments. High-throughput sequencing methods have made bacterial community profiling routine and affordable. But this has come at the expense of read length with most platforms covering 250 to 
$33600 \mathrm{bp}$ of the $\sim 1500 \mathrm{bp} 16 \mathrm{~S}$ rRNA gene, where increases in read length are generally

34 accompanied by decreases in read accuracy. Depending on the region sequenced, shorter

35 fragments give variable taxonomic and phylogenetic resolution (Claesson et al. 2010; Ghyselinck

36 et al. 2013; Schloss 2010) and fail to resolve differences outside the targeted region, which may

37 be ecologically relevant (Denef et al. 2010; Fitz-Gibbon et al. 2013; Moore et al. 1998).

39 The rise of the shorter fragment, high-throughput methods has also resulted in a lack of quality, 40 full-length reference sequences being deposited into reference databases, limiting our ability to 41 classify shorter reads from taxa that are underrepresented in these databases (Schloss et al. 2016).

42 Interpretation of 16S rRNA gene amplicon sequencing data is further confounded by PCR and 43 sequencing artefacts including chimeras, biased amplification and sequencing errors. Some of 44 these artefacts can be removed computationally (Schloss et al. 2011), but nevertheless lead to 45 errors that artificially inflate diversity estimates (Faith et al. 2013; Kunin et al. 2010; Lundberg et 46 al. 2013) and mislead analysis.

47

48 In this study, we have developed a method for sequencing near full-length $16 \mathrm{~S}$ rRNA sequences 49 on the high-throughput Illumina MiSeq platform. We provide proof of principle for the method by application to the skin microbiota with the reconstruction of high quality near full-length sequences. This method additionally provides the ability to remove putative chimeras and 52 amplification bias.

53 


\section{METHODS}

57

\section{Extraction of microbial DNA from foot skin}

59 DNA was extracted from skin swabs taken from the feet of 3 different healthy individuals. 12 samples were taken in total. Skin swabs were collected by swabbing either the ball or heel area of the left or right foot with a rayon swab moistened in a solution of $0.15 \mathrm{M} \mathrm{NaCl}$ and $0.1 \%$ Tween 20. The swab was rubbed firmly over the skin for approximately 30 seconds. Swab heads were cut into bead beating tubes, and DNA was extracted from the swabs using the BioStic DNA extraction kit (Mo-Bio), as per the manufacturers instructions. DNA was quantified on a Qubit with a HS-DNA assay (Life Technologies). Ethics approval for this study was given by the University of Technology Sydney Human Research Ethics Committee (approval number 2013000170), and participants provided written consent.

Preparation of short read 16S rRNA gene libraries for Illumina sequencing

Libraries $(n=12)$ of the V4 region of the 16S rRNA gene were prepared for Illumina sequencing from the microbial foot skin DNA samples using a modification of a previously published method (Caporaso et al. 2012). Briefly, samples were amplified using primers based on the Caporaso design (Caporaso et al. 2012), which were modified to include 8 nt rather than $12 \mathrm{nt}$

74 barcodes and include a barcode on both the forward and reverse primer. The V4 region was amplified from $500 \mathrm{pg}$ template DNA using 10 cycles of PCR with the modified Caporaso primers (V4_forward and V4_reverse), using different barcoded primer pairs for each sample

77 (Table S1). After removal of excess primer via a magnetic bead clean up the samples were 78 pooled and subjected to a further 20 cycles of PCR to enrich for amplicons containing the 
79 Illumina adapters, using primers Illumina_E_1 and Illumina_E_2 (Table S1). Pooling of

80 samples during the enrichment PCR allows for an assessment of the putative recombination rate,

81 by examining the rate of invalid barcode combinations that occur in the final paired end

82 sequencing data. The method for each PCR reaction is described in detail below.

83

84 PCRs were carried out with a Taq core PCR kit (Qiagen), under the following conditions. For 85 the initial 10 cycle PCR, reactions contained 1 x PCR buffer, 1 x Q solution (Qiagen), $250 \mu \mathrm{M}$ $86 \mathrm{dNTPs}, 0.5 \mu \mathrm{M}$ each of V4_forward and V4_reverse barcoded primers, 500 pg DNA template, 87 and 1.25 $\mathrm{U}$ Taq DNA polymerase in a $50 \mu \mathrm{l}$ reaction volume. Thermal cycling was carried out at $8895^{\circ} \mathrm{C}$ for 2 minutes, followed by 10 cycles of $95^{\circ} \mathrm{C}$ for 15 seconds, $50^{\circ} \mathrm{C}$ for 30 seconds and $8972^{\circ} \mathrm{C}$ for 90 seconds, followed by a final extension at $72^{\circ} \mathrm{C}$ for 5 minutes. After a magnetic bead 90 clean-up using 0.8 volume of SPRIselect beads (Beckman Coulter), the cleaned PCR reactions 91 were pooled and used as input for the second PCR reaction. This PCR contained $1 \times$ PCR buffer, $921 \times \mathrm{Q}$ solution (Qiagen), $250 \mu \mathrm{M}$ dNTPs, $0.25 \mu \mathrm{M}$ each of Illumina_E_1 and Illumina_E_2 93 primers (see Table S1), $31 \mu$ pooled PCR products from the first PCR, and 1.25 U Taq DNA 94 polymerase in a $50 \mu 1$ reaction volume. Thermal cycling was carried out at $95^{\circ} \mathrm{C}$ for 2 minutes, 95 followed by 20 cycles of $95^{\circ} \mathrm{C}$ for 15 seconds, $55^{\circ} \mathrm{C}$ for 30 seconds and $72^{\circ} \mathrm{C}$ for 90 seconds, 96 followed by a final extension at $72^{\circ} \mathrm{C}$ for 5 minutes. These PCR reactions were again cleaned 97 via a magnetic bead clean-up as above and run on an Agilent Bioanalyzer using a HS-DNA chip 98 to confirm the amplicon size and determine the concentration. Negative control PCRs were 99 included at all stages, and all PCR products were discarded if there was any evidence of a 100 product in the negative controls. 
102 The short read 16S rRNA libraries were sequenced using a Nano flow cell and a 500 cycle V2 kit

103 on an Illumina MiSeq, using custom primers as described previously (Caporaso et al. 2012).

104 This method will be referred to as "short sequencing" and data produced with this method as

105 "V4" data. Read pairs were merged with FLASH (Magoc \& Salzberg 2011) and de-multiplexed

106 using a new module implemented in a previously published version of PhyloSift (Darling et al.

107 2014).

108

109 Sequencing of near full-length 16S rRNA gene sequences on the Illumina MiSeq

110 We present a method to sequence near full-length 16S rRNA gene amplicons using Illumina 111 technology. The technique incorporates randomized molecular tags on both ends of individual 112 16S rRNA gene template molecules prior to PCR amplification. Copies of the templates are 113 fragmented and sequenced and the dual tag information is used to accurately re-assemble near 114 full-length 16S rRNA gene sequences. An overview of the method is shown in Figure 1, and a 115 detailed description is provided below.

116 Preparation of near full-length $16 S$ rRNA gene libraries for Illumina sequencing with unique 117 molecular tags on both ends

118 Primers for amplification of the 16S rRNA gene contained the 27F (Weisburg et al. 1991) or 119 1391R (Turner et al. 1999) bacterial primer sequences, an $8 \mathrm{nt}$ barcode sequence, a $10 \mathrm{nt}$ random 120 tag and partial Illumina PE adapter sequences (Figure 2, Table S1). The use of a 10 nt random 121 tag on both forward and reverse primers $(\sim 1$ million possible unique tags at each end, $\sim 1$ trillion 122 combinations) allowed us to uniquely tag each 16S rRNA gene molecule in our pool, by 123 modifying previously described tagging approaches (Faith et al. 2013; Lundberg et al. 2013). 
124 Template DNA was subjected to one cycle of annealing and extension with the forward primer

125 (long_forward, Table S1), followed by a magnetic bead clean up to remove excess primer, then

126 another cycle of annealing and extension with the reverse primer (long_reverse, Table S1),

127 followed by another magnetic bead clean up. The first PCR carries out extension of the 16S

128 rRNA gene from the forward primer, which uniquely tags different 16S rRNA gene templates in

129 the reaction. The second PCR uses extension products from the first PCR as a template to 130 produce molecules with unique tags at both ends. While the original 16S rRNA gene molecules

131 may also act as a template in the second PCR reaction, these products will only contain an

132 Illumina adapter at one end and will therefore not be amplified in the enrichment PCR. The

133 enrichment PCR (34 cycles) amplifies the tagged 16S rRNA gene molecule pool, using primers

134 that are complementary to the Illumina adapter sequences at the ends of each tagged 16S rRNA 135 gene molecule (primers PE_1 and PE_2, Table S1).

136

137 PCRs were carried out using the Taq PCR core kit (Qiagen), and differently barcoded primers

138 were used for each sample. Reactions contained approximately 500 pg DNA template, $0.25 \mu \mathrm{M}$

139 long_forward primer, $250 \mu \mathrm{M}$ dNTPs, 1 x PCR buffer, 1 x Q solution, and 1.25 U Taq

140 polymerase in a $50 \mu \mathrm{l}$ volume. Cycle conditions were $95^{\circ} \mathrm{C}$ for 1 minute, $50^{\circ} \mathrm{C}$ for 2 minutes

141 then $72^{\circ} \mathrm{C}$ for 3 minutes. This allows extension of the $16 \mathrm{~S}$ rRNA gene from the forward primer,

142 which uniquely tags the forward end of each $16 \mathrm{~S}$ rRNA gene molecule in the reaction. PCR

143 reactions were then subjected to a magnetic bead clean up using 0.6 volumes of SPRIselect beads

144 (Beckman Coulter) as per the manufacturer's instructions, except that the DNA was eluted in 35

$145 \mu 1$ nuclease free water. The second PCR was set up as described above, except that $0.25 \mu \mathrm{M}$ of

146 the long_reverse primer was used and the template was $31 \mu \mathrm{l}$ of the bead-cleaned first round 
147 annealing and extension reaction. Only 31 of the $35 \mu$ of bead cleaned first round PCR was

148 used, to prevent contamination with the magnetic beads. Cycling conditions were as in the 149 previous step: $95^{\circ} \mathrm{C}$ for 1 minute, $50^{\circ} \mathrm{C}$ for 2 minutes and $72^{\circ} \mathrm{C}$ for 3 minutes. During this

150 second reaction, the uniquely tagged extension products from the first reaction act as the 151 template to produce $16 \mathrm{~S}$ rRNA gene molecules with unique tags on the forward and reverse 152 ends. This was followed by another magnetic bead clean up, as described above, and the output 153 of this step was used as a template for the final PCR reaction. The final enrichment PCR 154 reaction contained $0.5 \mu \mathrm{M}$ of each PE_1 and PE_ 2 primers, $250 \mu \mathrm{M}$ dNTPs, 1 x PCR buffer, $1 \mathrm{x}$ 155 Q solution, $31 \mu$ template (from the bead clean up) and $1.25 \mathrm{U}$ Taq polymerase in a $50 \mu \mathrm{l}$ 156 volume. Cycling conditions were $95^{\circ} \mathrm{C}$ for 2 minutes, followed by 34 cycles of $95^{\circ} \mathrm{C}$ for 1 157 minute, $58^{\circ} \mathrm{C}$ for 30 seconds, $72^{\circ} \mathrm{C}$ for 2 minutes, and a final extension of $72^{\circ} \mathrm{C}$ for 5 minutes. 158 PCRs were again subjected to a magnetic bead clean up as described above, before being 159 analysed using a high-sensitivity DNA chip on a Bioanalyser (Agilent) to determine amplicon 160 size and concentration. Negative control PCRs were included at all stages, and all PCR products 161 were discarded if there was any evidence of a product in the negative controls. This was 162 assessed via Bioanalyser traces from HS-DNA chips, although we acknowledge it is possible that 163 products below the limit of detection may still have been present.

164

165

Tagmentation of near full-length $16 \mathrm{~S}$ rRNA gene amplicon libraries

166 The uniquely tagged, near full-length 16S rRNA gene PCR amplicons were subjected to 167 tagmentation. The tagmentation procedure utilises a transposase to simultaneously fragment the 168 DNA while adding an adapter sequence for use on the Illumina platform. Tagmentation was 169 carried out using the Nextera XT kit as per the manufacturer's instructions, with the exception of 
170 the PCR amplification step. Here, we split the tagmentation reaction into two and carried out

171 two separate PCRs at half the volume specified in the kit (where normally only one PCR is

172 carried out). Each PCR reaction contained a combination of one of the Illumina provided

173 Nextera XT PCR primers and one of the primers from the enrichment PCR above, so as to

174 amplify only those fragments of interest; specifically, we combined primers PE_1 and an

175 Illumina Index 1 primer (N706) in one PCR reaction and PE_ 2 and an Illumina Index 2 primer

176 (S504) in the second. We aimed to produce a pool of DNA fragments with either the PE_1

177 (forward end of the 16S rRNA gene amplicons) or PE_2 (reverse end of the 16S rRNA gene

178 amplicons) sequences on one end and the $i 7$ or i5 Illumina adapters (added to an internal region

179 of the amplicon during the tagmentation reaction) at the other end, respectively. Each $25 \mu 1$ PCR

180 reaction contained 1 x Nextera PCR master mix with either $2.5 \mu$ N706 Nextera index primer

181 and PE_1 primer at $1 \mu \mathrm{M}$, or $2.5 \mu \mathrm{S} \mathrm{S504} \mathrm{index} \mathrm{primer} \mathrm{and} \mathrm{PE} 2$ primer at $1 \mu \mathrm{M}$, and $12.5 \mu 1$ of

182 the tagmentation reaction. PCR reactions were carried out as follows; $72^{\circ} \mathrm{C}$ for 3 minutes, $95^{\circ} \mathrm{C}$

183 for 30 seconds, then 12 cycles of $95^{\circ} \mathrm{C}$ for 10 seconds, $60^{\circ} \mathrm{C}$ for 30 seconds and $72^{\circ} \mathrm{C}$ for 1

184 minute, followed by a final extension at $72^{\circ} \mathrm{C}$ for 5 minutes. These two PCR reactions provided

185 a pool of fragments from across the 16S rRNA gene, which along with the full-length amplicons,

186 could be paired end sequenced on the MiSeq. PCR products from the tagmentation reaction

187 were cleaned using 0.6 volumes of SPRIselect beads according the manufacturer's instructions,

188 to remove fragments smaller than $400 \mathrm{bp}$. This step was necessary to achieve the desired range

189 of fragment sizes $(\sim 400-1500 \mathrm{bp})$ to ensure adequate coverage across the full span of the 16S

190 rRNA gene amplicon.

191

192 Sequencing of near full-length and tagmented 16S rRNA gene amplicon libraries 
193 The molarity of both near full-length and tagmented 16S rRNA gene amplicon libraries was

194 measured via an Agilent Bioanalyser High Sensitivity DNA chip. For the tagmented libraries,

195 molarity was calculated based on the 400-1000 bp range. Tagmented libraries were pooled at

196 equal molarity and combined with the full-length amplicons at a molar ratio of $\sim 7: 1$

197 (tagmentation pool: full length amplicons), with the tagmented pool at $\sim 2.6 \mathrm{pM}$ for loading. The

198 pool was sequenced with 2 x 250 paired end reads, on a MiSeq Nano flow cell.

Reconstructing full-length $16 S$ rRNA gene sequences from tagged Illumina reads

200 Sequencing produced data from two kinds of fragments, those that span the entire 16S rRNA

201 gene (end+end fragments) and those that contained either the forward or reverse end of the gene

202 at one end with a region in the middle of the gene at the other end (end+internal fragments).

203 Sequences from end+end fragments encoded a pairing of molecular tags and sample barcodes.

204 Sequences were assigned to bins of original 16S rRNA gene progenitor molecules via the unique 205 tags at either end of the molecule and were re-assembled to provide near full-length 16S rRNA 206 gene sequences. Figure 3 shows an overview of the process.

207

208 To assign sequences to samples, the two $8 \mathrm{nt}$ sample barcode regions were matched against the 209 collection of known sample barcodes with up to one mismatch tolerated in each 8 nt barcode.

210 Because internal regions of the 16S rRNA gene sequence might match a sample barcode, all

211 reads with a potential sample barcode match were then screened for the presence of the proximal 212 or distal 16S rRNA gene primer annealing sequence downstream from the sample barcode. 213 Reads lacking a known sample barcode or the primer annealing sequence in one end were 214 presumed to derive from an end+internal fragment. 
216 Consensus molecular tags and elimination of recombinants: Due to sequencing error, the reads

217 derived from the same template molecule may have had slightly different $10 \mathrm{nt}$ random tagging 218 sequences. To estimate the original $10 \mathrm{nt}$ random tag sequences of tagged template molecules the 219 UCLUST (Edgar 2010; Edgar 2013) algorithm was applied to identify clusters of matching 220 random tag sequences at $>89 \%$ identity (e.g. 1 out of 10 bases mismatch) and to report the 221 consensus sequences of these clusters. Clusters of molecular tags in the end+end fragments (the 222 clustered sequences consisting of both $10 \mathrm{nt}$ random tags, both $8 \mathrm{nt}$ sample barcodes, and the first $22314 \mathrm{nt}$ of the $16 \mathrm{~S}$ rRNA gene amplicon sequence in each read) were first identified. This was

224 followed by the identification of the highest abundance cluster with the same combination of 10 225 nt random tags (one from either end) and discarding of any cluster containing one or both $10 \mathrm{nt}$ 226 random tags that were found in a different, more abundant cluster. This step aimed to identify 227 and discard combinations of molecular tags that arose due to in vitro recombination. 228 Recombinant forms are likely to be at lower abundance than the parental templates.

229

230 Finally, molecular tags from the entire set of reads (end+end and end+internal) were matched 231 against the collection of consensus sequences and the reads were grouped into clusters for later 232 assembly.

233

234 Assembly of read clusters: Each read cluster contained reads that, with high probability, 235 originated from the same template molecule. A de novo assembly algorithm was applied on the 236 read cluster to reconstruct as much of the original template molecule as possible. The reads were 237 assembled using a version of the A5 pipeline called A5-miseq (Coil et al. 2015) that has been 238 modified to support assembly of reads up to $500 \mathrm{nt}$ long and to trim out adapter sequence from 
239 reads instead of discarding reads containing adapter sequence. Only the first two stages of the

240 A5-miseq pipeline were applied, involving adapter trimming, quality trimming, error correction,

241 and contig assembly.

242

243 This method of amplifying, sequencing and assembling 16S rRNA gene sequences will be

244 referred to as "Long-16S" and data produced with this method as "Long-16S" data, from here on 245 in.

246

247

Assessment of assembled Long-16S sequence quality

248 The accuracy of the base calls was estimated by calculating PHRED scale quality scores (Ewing

249 et al. 1998) using samtools ( $\mathrm{Li}$ et al. 2009). Briefly, the reads present in each assembled 250 molecular tag cluster were mapped back to the assembled contigs using BWA MEM (Li 2013).

251 From the mapped reads, a consensus FastQ sequence was called using samtools, bcftools, and 252 vcfutils.pl (Li et al. 2009). The quality scores in the resulting FastQ file were then used for 253 subsequent quality analysis and visualization.

254

255 Removal of chimeras in cluster assemblies

256 Putative chimeras were identified in end+end reads as described above; this permitted estimation 257 of the overall recombination rate and the frequency of recombinant fragments relative to full258 length fragments for each cluster. However, it was not possible to directly identify end+internal 259 reads derived from a chimeric fragment using molecular tags, as some of these reads contained a 260 molecular tag that matched an original template cluster. Erroneous signal from these reads was 261 eliminated in two ways, both of which depended on reads derived from the recombinant form 
262 existing at lower abundance in the sequence data. First, during the initial assembly process, k-

263 mer error correction and consensus generation eliminated differences in the sequence present in

264 low abundance chimeric reads. Second, in cases where the cluster assembly contained multiple

265 contigs, the depth of coverage of contigs was used to identify and remove contigs at much lower

266 abundance than the dominant contigs in the cluster. For the present work we removed any

267 contigs with an average coverage that was 10-fold lower than that of the highest abundance

268 contig. Future work could use information derived from the end+end sequences to estimate the

269 expected fraction of recombinant reads in a cluster and use this to aid the process of eliminating

270 chimera-derived contigs or to identify clusters for which recombinant elimination may not be

271 possible.

272

\section{Analysis of V4 and Long-16S data}

274

275 Both V4 and Long-16S data generated from the 12 skin samples were analysed using the 276 software package QIIME (Caporaso et al. 2010). For comparison, the corresponding V4 region 277 was extracted from the Long-16S sequences (which we will refer to as extracted-V4). Only 278 those Long-16S sequences that were $>1300 \mathrm{nt}$ in length were included in the downstream 279 analysis. V4 sequences were initially quality filtered using the default settings, with the 280 exception of sequence length, which was altered to remove sequences less than $240 \mathrm{nt}$ and longer 281 than $260 \mathrm{nt}$. V4 sequences were additionally checked for the presence of chimeras using the 282 UCHIME (Edgar et al. 2011) method, both against a reference database (RDP Classifier 16S 283 training set No9, accessed from $\underline{\text { https://sourceforge.net/projects/rdp- }}$ 284 classifier/files/RDP Classifier_TrainingData/), as well as using the dataset itself as the reference. 
285 Long-16S sequences were quality filtered using default settings and excluding sequences longer 286 than $1400 \mathrm{nt}$. Quality filtered sequences from the V4, Long-16S and extracted-V4 datasets were 287 then combined, and sequences were assigned to OTUs using the closed reference picking 288 method, which assigns sequences to pre-clustered OTUs at $97 \%$ similarity from the chimera 289 filtered Greengenes database (DeSantis et al. 2006; McDonald et al. 2012). OTUs with less than 2902 sequences were filtered from the OTU table. Taxonomy was determined based on membership 291 to the database of pre-clustered OTUs, and the relative abundance of taxa at different levels was 292 generated using the summarize_taxa.py script. Differences in abundance of taxa at phylum and 293 genera level between the V4 and Long-16S data were tested for significance using the 294 group_significance.py script with taxa summary biom tables as input and using the Kruskal295 Wallis test with the Benjamin Hochberg FDR correction for multiple testing. 296

\section{Assessment of bias reduction using unique molecular tags}

298 The use of molecular tagging has previously been shown to reduce the effect of PCR bias in RNA-seq data, for better quantitative assessment of sequences from the original samples (Islam

300

301

302

303

304

305

306

307 et al. 2014). Assuming that each uniquely tagged 16S rRNA gene molecule from our skin samples was present at the same abundance as all other uniquely tagged molecules (i.e. 1 copy of each) and that unbiased amplification would result in an equal abundance of each cluster, we estimated the amount of biased amplification that occurred during PCR by comparing the differences in the abundance of end+end sequence clusters. 


\section{RESULTS}

310 Near full-length 16S rRNA gene sequences from an Illumina MiSeq generated by

\section{1 molecular tagging}

312 Sequencing of both the full-length and the tagmented amplicon pools was successful with a 313 cluster density of $400-500 \mathrm{k} / \mathrm{mm} 2$ and 832,293 read pairs. Higher clustering and sequencing 314 output may be possible by optimising the loading concentration and ratio of end+end and 315 end+internal pools. Clustering of end+end reads resulted in 5085 clusters. Of these, 2265 $316(44.6 \%)$ were deemed to be putative recombinant clusters, with predicted parental templates on 317 average 29 times more abundant than putative recombinants (Figure 4). Putative recombinant 318 end+end sequences represented 4378 of the total 42715 sequences in the end+end read pool, 319 indicating an average recombination rate of $10.2 \%$ among all samples. After binning and 320 assembly of end+end and end+internal read clusters, $230416 \mathrm{~S}$ rRNA gene sequences were 321 assembled from 558,053 Illumina read pairs. Sequence lengths ranged from 449 to $1372 \mathrm{nt}$ (full-

322 length), and $\sim 70 \%$ of these were greater than $1300 \mathrm{nt}$. The assembly of sequences with less than 323 the expected length (i.e. those $400-1300 \mathrm{bp}$ ) is possibly due to a lack of coverage across the 324 internal regions for some end+end clusters. The range of sequence lengths generated is shown in 325 Figure 5.

326

327 Assembled sequences had consistently high quality scores across their length, with the per site 328 average estimated PHRED quality scores at each position ranging from 54.0 - 89.5 (median 329 68.0) (Figure 6a). This indicated estimated base-calling accuracies of greater than $99.999 \%$ at 330 each position of the assembled $16 \mathrm{~S}$ rRNA gene sequences. We note that errors due to base 
331 misincoporations that occur during early cycles of the enrichment PCR cannot be directly

332 measured with this method, therefore these estimates of consensus accuracy may overestimate

333 the true accuracy of the reconstructed 16S rRNA genes. Higher qualities for the Long-16S

334 sequences were associated with higher coverage, which is particularly apparent at each end of 335 the reconstructed sequences (up to 200 and beyond $1200 \mathrm{nt}$ ), which were associated with one 336 read from every read pair (end+internal or end+end) in the data set (Figure 6b).

337

\section{Short sequencing of the $16 \mathrm{~S}$ rRNA gene $\mathrm{V} 4$ region}

339 A total of 296,864 paired end 16S rRNA gene V4 sequences were generated from the 12 skin 340 samples. Of these sequences, 11,240 could not be assigned to a sample due to invalid forward 341 and reverse barcode combinations (e.g. combinations which were never assigned to a sample), 342 indicating an in-vitro recombination rate of at least 3.8\%, although a small proportion of this 343 may be due to cross-contamination of barcoded primers (Kircher et al. 2012). These sequences

344 were removed from the dataset. We note that in-vitro recombination could also create barcode 345 combinations that would match a valid sample and therefore be undetectable recombination 346 events. In contrast, when attempting to detect recombination products using the chimera 347 detection software UCHIME (as implemented in QIIME), only $0.05 \%$ of the sequences were 348 flagged as chimeric when compared against a reference database (SILVA) and $0.2 \%$ when using 349 the dataset itself as the reference. This highlights the difficulties of using software alone to 350 detect recombination products from PCR in the absence of sample barcode and molecular tag 351 information. Sequences that were flagged as chimeric using UCHIME, which had not been 352 identified as chimeric based on sample barcode combinations (as described above) were also 353 removed from the dataset. 
355 Assembled near full-length 16S rRNA gene sequences produce data consistent with short 356 read sequencing.

357 Taxonomy, as assigned in QIIME, was similar to previous reports for skin communities, 358 dominated by Firmicutes, Actinobacteria, and Proteobacteria. Long-16S and extracted-V4 OTUs 359 showed the same broad taxonomic distribution as the V4 sequence data (Figure 7). There was a 360 small decrease in the representation of Firmicutes and an increase in the representation of 361 Actinobacteria and Proteobacteria (Figure 7), however these differences were not significant 362 (Kruskal-Wallis with Benjamin-Hochberg FDR correction for multiple testing, $\mathrm{p}>0.05$ ). 363 Similar taxonomic assignments between the different sequencing methods were also observed at 364 the level of genus, with communities dominated by Staphylococcus, followed by 365 Corynebacterium, Enhydrobacter and Acinetobacter. The Corynebacterium genus had an 366 increased representation in the full-length data set as compared to the V4 data, which likely 367 accounts for the observed difference in representation for the Actinobacteria phyla, but as above, 368 this difference was not significant (Kruskal-Wallis with Benjamin-Hochberg FDR correction for 369 multiple testing, $\mathrm{p}>0.05)$.

370

371 Comparison at the OTU level: Of the OTUs clustered at 97\% similarity from the 12 libraries of 372 Long-16S sequence data, an average of $22.7 \%( \pm 15.6)$ were also found in matched sample V4 373 data that was clustered in the same way. This disparity is likely due to comparing OTUs of 374 sequences of different lengths and the way in which OTUs are defined in QIIME. Sequences are 375 assigned to OTUs by clustering (UCLUST method) against a database of full-length 376 representative sequences (from the Greengenes database (DeSantis et al. 2006)) which are at 
377 most $97 \%$ similar to each other and are used as centroids for each cluster. Sequences that are

$37897 \%$ similar across the full $16 \mathrm{~S}$ rRNA gene may be more or less than $97 \%$ similar in the V4

379 region only, since different regions of the 16S rRNA gene evolve at different rates (Schloss

380 2010). As such, V4 sequences will be assigned to the OTU cluster from the first representative

381 sequence in the database that is at least $97 \%$ similar within the V4 region, while longer

382 sequences that contain an identical V4 region but are not $97 \%$ similar to the same cluster 383 centroid will be assigned to a different OTU. We therefore analysed OTUs clustered from the V4

384 region of the Long-16S sequences (extracted-V4 sequences) in comparison to OTUs clustered 385 from the V4 data to determine whether we had captured similar OTUs with the V4 and Long-16S 386 sequencing methods. In this case $83.7 \%( \pm 15.9)$ of extracted-V4 OTUs were shared with the 387 matched sample V4 OTUs (Table S2). Although fewer sequences were present in the Long-16S 388 data set, yielding many fewer OTUs overall, the data indicates that the newly developed method 389 gives broadly congruent community profiles with respect to taxonomy and OTU clustering.

390

391 Assessment of bias reduction using unique molecular tags

392 We estimated the amount of biased amplification that occurred during PCR by comparing the 393 differences in the abundance of end+end sequence clusters. The average abundance was 394 calculated from all clusters, and the relative mean error was 2.08 , or 1.81 if singleton clusters 395 (possible recombinants) were excluded. This indicates a standard deviation of approximately 2 396 times the average across the dataset under the particular amplification conditions used here. 397 Figure 8 shows the distribution of the estimated amplification bias, which ranges from 0.06 to $\sim$ 39832 times the average cluster abundance. This potential bias is eliminated by considering each 399 assembled sequence cluster as having a count of 1 . 


\section{Discussion}

402 We have presented a method for sequencing near full-length 16S rRNA gene sequences on the 403 high throughput Illumina MiSeq platform. This method utilizes tagging of individual 16S rRNA 404 gene template molecules with unique, random sequences (tags), an approach that has been used 405 previously to generate consensus sequences from short read data (Faith et al. 2013; Lundberg et 406 al. 2013). These previous approaches targeted individual variable regions rather than sequencing 407 of the whole gene, and incorporated tags at only one end of the amplicon (Faith et al. 2013) or 408 tags too short (Lundberg et al. 2013) to permit the assembly and chimera filtering techniques 409 proposed here. This new method incorporates randomized molecular tags on both ends of 410 individual 16S rRNA gene template molecules prior to PCR amplification and uses this dual tag 411 information to reconstruct near full-length 16S rRNA gene sequences and remove putative 412 chimeras.

414 We assembled 2304 16S rRNA gene sequences in a single MiSeq Nano run, 1604 of which were 415 longer than $1300 \mathrm{bp}$. There are at least two factors that could contribute to the assembly of 416 templates $<1300 \mathrm{bp}$. The first is a lack of read coverage throughout the full sequence, causing 417 fragmentation in the 16S rRNA gene assembly. A second likely contributing factor is collisions 418 in molecular tags, e.g. when different templates receive the same molecular tag on one (or both) 419 end by chance. Exact matches are expected to be rare when sampling 5000 items with 420 replacement from a pool of 1 million (the number of random 10-mers). However, when 421 mismatches are permitted in molecular tags to accommodate sequencing error, the expected rate 422 of collisions increases. Distinguishing sequencing errors in molecular tags from tag collisions is 
423 an area for future research. Finally, a third factor that could contribute to these short reads is that

424 although differences in the abundance of molecular tag pairs enable true full length templates to

425 be distinguished from recombinant forms, our protocol does not provide direct information to

426 distinguish whether the end+internal reads derive from a true template or a recombinant form.

427 Therefore the collection of end+internal reads associated with an end+end template may contain

428 contaminating recombinant reads. When these contaminating reads are at sufficiently high

429 abundance, and are sufficiently divergent from the other end+internal reads they can cause

430 assembly fragmentation. Our data processing scripts (available in github) include steps to

431 eliminate contigs that have very low relative coverage within a molecular tag cluster, but the

432 assembly fragmentation caused by the putative contaminant reads could remain.

433

434 While only a small amount of data were analysed here, we have provided proof of principle that 435 this method is capable of generating many high quality, near full-length 16S rRNA gene 436 sequences, using one of the most cost effective and widely available high-throughput sequencing 437 platforms. Assuming linear scaling, the method could yield up to 80,000 full-length 16S rRNA 438 gene sequences on a 600 cycle MiSeq v3 kit, while a HiSeq 2500 might generate up to 480,000 439 near full-length 16S rRNA gene sequences in a single "rapid run" lane. This potentially places 440 the reagent cost per 16S rRNA gene sequence in the region of US\$0.006-\$0.025, making the cost 441 of producing these sequences much lower than traditional Sanger sequencing ( $\sim$ US $\$ 8$ per 442 sequence) (Schloss et al. 2016). Protocols for the generation of near full-length 16S rRNA gene 443 sequences on other platforms have also been described (Benitez-Paez et al. 2016; Fichot \& 444 Norman 2013; Schloss et al. 2016; Singer et al. 2016), but at present they cannot match the high 445 quality, throughput and cost efficiency of Illumina platforms. 
447 This method is more expensive and has lower throughput than sequencing short regions of the

448 16S rRNA gene, but the cost could be justified where the increased resolution afforded by long

449 16S rRNA gene sequences is required. Accurate classification of short reads is dependent on the 450 completeness of reference databases and training sequences used (Werner et al. 2012), and the 451 high quality sequences generated with this method could be particularly useful for providing 452 reference sequences for environments or taxa that are poorly represented in the current databases.

453 Several recent studies utilized traditional cloning and Sanger sequencing of near full-length 16S 454 rRNA genes in combination with high throughput short read sequencing for this purpose 455 (Chaves-Moreno et al. 2015; Dewhirst et al. 2015; Hund et al. 2015). There is also growing 456 interest in the ability to resolve species and strain level differences from microbiota data (Eren et 457 al. 2015; Greenblum et al. 2015; Kraal et al. 2014; Luo et al. 2015; Tikhonov et al. 2015), and 458 the additional information obtained with accurate near full-length 16S rRNA gene sequencing 459 could be used to better identify putative strains of bacteria between and within samples.

460

461 We estimated a 100-fold reduction in average error rate with our method compared to paired-end 462 sequencing of short regions. While estimated accuracy was very high, the presence of errors 463 introduced by base misincorporation during PCR cannot be directly assessed in this dataset and 464 will still be present. We chose to use a standard Taq polymerase over a high-fidelity polymerase, 465 as preliminary experiments indicated an extremely high rate of recombination with the high466 fidelity enzyme tested. Based on published estimates of the Taq polymerase error rate $\left(\sim 3 \times 10^{-}\right.$ $467{ }^{5}$ ) (McInerney et al. 2014), we expect around 1 error per 10 tagged templates to occur prior to 468 PCR enrichment. Errors that occurred during the enrichment PCR after tagging and after 
469 tagmentation were potentially corrected via the consensus sequence, depending on how early in

470 the PCR individual errors occurred. The use of a mock community would allow for more robust

471 testing of PCR and sequencing error rates, and other potential sources of error common to all

472 16S rRNA gene amplicon sequencing protocols (e.g. PCR conditions and priming regions used).

473

474 Artifacts generated through the PCR process, including chimera formation and biased 475 amplification of a subset of templates have been acknowledged to be a problem in surveys of 476 microbial communities for some time (Judo et al. 1998; Kopczynski et al. 1994; Liesack et al. 477 1991; Polz \& Cavanaugh 1998). Despite attempts to minimize these effects via tuning of 478 experimental parameters (Fonseca et al. 2012; Judo et al. 1998; Smyth et al. 2010) or 479 computational detection (Ashelford et al. 2005; Edgar et al. 2011; Haas et al. 2011) these 480 artifacts remain and may confound data analysis. The use of a dual tag system as demonstrated 481 here offers an alternative signal for both the removal of putative chimeras, and the correction of 482 PCR bias (Islam et al. 2014). Using this method, we were able to remove a large proportion of 483 putative chimeric sequences from the dataset and estimate the degree of bias (Figure 8). Because 484 the abundance profiles of the reconstructed near full-length $16 \mathrm{~S}$ rRNA gene sequences work on 485 the assumption that each tagged template was originally present as a single copy, this method 486 provides a way to minimise PCR bias when applied to microbial communities.

\section{Conclusion}

489 We have provided proof of principle that this method enables the generation of large numbers of 490 high quality, near full-length 16S rRNA gene sequences. We note that the method of dual 491 molecular tagging could be applied to any sequencing platform and any amplicon target to 
492 enhance chimera removal and reduce amplification bias and base calling error. This is valuable

493 for the expansion of current databases with high quality, near full-length reference sequences.

494 Additionally, in conjunction with new algorithms (Eren et al. 2015; Tikhonov et al. 2015), this

495 method could facilitate a finer understanding of population dynamics in microbial ecosystems.

496

497 ACCESSIONS

498 Raw read data are available from SRA accession SRX655489. Assembled 16S rRNA gene

499 sequences have been provided as a Supplementary data set, Long-16S.fasta

500

501 Software automating the process of sample demultiplexing, molecular tag clustering, and

502 amplicon assembly is available from http://github.com/koadman/longas.

503

\section{ACKNOWLEDGMENTS}

505 We thank Josh Quick for advice on configuring the MiSeq to cluster long fragments, Paul

506 Worden for assistance operating the MiSeq instrument, and Torsten Thomas for helpful

507 discussions and suggestions during manuscript preparation.

508

509

510

511

512

513

514

515

516

517

518

519

Ashelford KE, Chuzhanova NA, Fry JC, Jones AJ, and Weightman AJ. 2005. At least 1 in 20 16S rRNA sequence records currently held in public repositories is estimated to contain substantial anomalies. Appl Environ Microbiol 71:77247736. 10.1128/AEM.71.12.7724-7736.2005

Benitez-Paez A, Portune KJ, and Sanz Y. 2016. Species-level resolution of 16S rRNA gene amplicons sequenced through the MinION portable nanopore sequencer. Gigascience 5:4. 10.1186/s13742-016-0111-z

Caporaso JG, Kuczynski J, Stombaugh J, Bittinger K, Bushman FD, Costello EK, Fierer N, Pena AG, Goodrich JK, Gordon JI, Huttley GA, Kelley ST, Knights D, Koenig JE, Ley RE, Lozupone CA, McDonald D, Muegge BD, Pirrung M, Reeder J, 
527

528

529

530

531

532

533

534

535

536

537

538

539

540

541

542

543

544

545

546

547

548

549

550

551

552

553

554

555

556

557

558

559

560

561

562

563

564

565

Sevinsky JR, Turnbaugh PJ, Walters WA, Widmann J, Yatsunenko T, Zaneveld J, and Knight R. 2010. QIIME allows analysis of high-throughput community sequencing data. Nat Methods 7:335-336. 10.1038/nmeth.f.303

Caporaso JG, Lauber CL, Walters WA, Berg-Lyons D, Huntley J, Fierer N, Owens SM, Betley J, Fraser L, Bauer M, Gormley N, Gilbert JA, Smith G, and Knight R. 2012. Ultra-high-throughput microbial community analysis on the Illumina HiSeq and MiSeq platforms. ISME J 6:1621-1624. 10.1038/ismej.2012.8

Chaves-Moreno D, Plumeier I, Kahl S, Krismer B, Peschel A, Oxley AP, Jauregui R, and Pieper DH. 2015. The microbial community structure of the cotton rat nose. Environ Microbiol Rep 7:929-935. 10.1111/1758-2229.12334

Claesson MJ, Wang Q, O'Sullivan O, Greene-Diniz R, Cole JR, Ross RP, and O'Toole PW. 2010. Comparison of two next-generation sequencing technologies for resolving highly complex microbiota composition using tandem variable $16 \mathrm{~S}$ rRNA gene regions. Nucleic Acids Res 38:e200. 10.1093/nar/gkq873

Coil D, Jospin G, and Darling AE. 2015. A5-miseq: an updated pipeline to assemble microbial genomes from Illumina MiSeq data. Bioinformatics 31:587-589. 10.1093/bioinformatics/btu661

Darling AE, Jospin G, Lowe E, Matsen FAt, Bik HM, and Eisen JA. 2014. PhyloSift: phylogenetic analysis of genomes and metagenomes. PeerJ 2:e243. 10.7717/peerj.243

Denef VJ, Kalnejais LH, Mueller RS, Wilmes P, Baker BJ, Thomas BC, VerBerkmoes NC, Hettich RL, and Banfield JF. 2010. Proteogenomic basis for ecological divergence of closely related bacteria in natural acidophilic microbial communities. Proc Natl Acad Sci U S A 107:2383-2390. 10.1073/pnas.0907041107

DeSantis TZ, Hugenholtz P, Larsen N, Rojas M, Brodie EL, Keller K, Huber T, Dalevi D, Hu P, and Andersen GL. 2006. Greengenes, a chimera-checked 16S rRNA gene database and workbench compatible with ARB. Appl Environ Microbiol 72:50695072. 10.1128/AEM.03006-05

Dewhirst FE, Klein EA, Bennett ML, Croft JM, Harris SJ, and Marshall-Jones ZV. 2015. The feline oral microbiome: a provisional 16S rRNA gene based taxonomy with full-length reference sequences. Vet Microbiol 175:294-303. 10.1016/j.vetmic.2014.11.019

Edgar RC. 2010. Search and clustering orders of magnitude faster than BLAST. Bioinformatics 26:2460-2461. 10.1093/bioinformatics/btq461

Edgar RC. 2013. UPARSE: highly accurate OTU sequences from microbial amplicon reads. Nat Methods 10:996-998. 10.1038/nmeth.2604

Edgar RC, Haas BJ, Clemente JC, Quince C, and Knight R. 2011. UCHIME improves sensitivity and speed of chimera detection. Bioinformatics 27:2194-2200. 10.1093/bioinformatics/btr381

Eren AM, Morrison HG, Lescault PJ, Reveillaud J, Vineis JH, and Sogin ML. 2015. Minimum entropy decomposition: unsupervised oligotyping for sensitive partitioning of high-throughput marker gene sequences. ISME J 9:968-979. 10.1038/ismej.2014.195

Ewing B, Hillier L, Wendl MC, and Green P. 1998. Base-calling of automated sequencer traces using phred. I. Accuracy assessment. Genome Res 8:175-185. 
566 Faith JJ, Guruge JL, Charbonneau M, Subramanian S, Seedorf H, Goodman AL,

567

568

569

570

571

572

573

574

575

576

577

578

579

580

581

582

583

584

585

586

587

588

589

590

591

592

593

594

595

596

597

598

599

600

601

602

603

604

605

606

607

608

609

610 Clemente JC, Knight R, Heath AC, Leibel RL, Rosenbaum M, and Gordon JI. 2013. The long-term stability of the human gut microbiota. Science 341:1237439. 10.1126/science.1237439

Fichot EB, and Norman RS. 2013. Microbial phylogenetic profiling with the Pacific Biosciences sequencing platform. Microbiome 1:10. 10.1186/2049-2618-1-10

Fitz-Gibbon S, Tomida S, Chiu BH, Nguyen L, Du C, Liu MH, Elashoff D, Erfe MC, Loncaric A, Kim J, Modlin RL, Miller JF, Sodergren E, Craft N, Weinstock GM, and Li HY. 2013. Propionibacterium acnes Strain Populations in the Human Skin Microbiome Associated with Acne. Journal of Investigative Dermatology 133:2152-2160. Doi 10.1038/Jid.2013.21

Fonseca VG, Nichols B, Lallias D, Quince C, Carvalho GR, Power DM, and Creer S. 2012. Sample richness and genetic diversity as drivers of chimera formation in nSSU metagenetic analyses. Nucleic Acids Res 40:e66. 10.1093/nar/gks002

Ghyselinck J, Pfeiffer S, Heylen K, Sessitsch A, and De Vos P. 2013. The effect of primer choice and short read sequences on the outcome of 16S rRNA gene based diversity studies. PLoS One 8:e71360. 10.1371/journal.pone.0071360

Greenblum S, Carr R, and Borenstein E. 2015. Extensive strain-level copy-number variation across human gut microbiome species. Cell 160:583-594. 10.1016/j.cell.2014.12.038

Haas BJ, Gevers D, Earl AM, Feldgarden M, Ward DV, Giannoukos G, Ciulla D, Tabbaa D, Highlander SK, Sodergren E, Methe B, DeSantis TZ, Human Microbiome C, Petrosino JF, Knight R, and Birren BW. 2011. Chimeric 16S rRNA sequence formation and detection in Sanger and 454-pyrosequenced PCR amplicons. Genome Res 21:494-504. 10.1101/gr.112730.110

Hund A, Dzieciol M, Schmitz-Esser S, and Wittek T. 2015. Characterization of mucosaassociated bacterial communities in abomasal ulcers by pyrosequencing. Vet Microbiol 177:132-141. 10.1016/j.vetmic.2015.02.023

Islam S, Zeisel A, Joost S, La Manno G, Zajac P, Kasper M, Lonnerberg P, and Linnarsson S. 2014. Quantitative single-cell RNA-seq with unique molecular identifiers. Nat Methods 11:163-166. 10.1038/nmeth.2772

Judo MS, Wedel AB, and Wilson C. 1998. Stimulation and suppression of PCRmediated recombination. Nucleic Acids Res 26:1819-1825.

Kircher M, Sawyer S, and Meyer M. 2012. Double indexing overcomes inaccuracies in multiplex sequencing on the Illumina platform. Nucleic Acids Res 40:e3. 10.1093/nar/gkr771

Kopczynski ED, Bateson MM, and Ward DM. 1994. Recognition of chimeric smallsubunit ribosomal DNAs composed of genes from uncultivated microorganisms. Appl Environ Microbiol 60:746-748.

Kraal L, Abubucker S, Kota K, Fischbach MA, and Mitreva M. 2014. The prevalence of species and strains in the human microbiome: a resource for experimental efforts. PLoS One 9:e97279. 10.1371/journal.pone.0097279

Kunin V, Engelbrektson A, Ochman H, and Hugenholtz P. 2010. Wrinkles in the rare biosphere: pyrosequencing errors can lead to artificial inflation of diversity estimates. Environ Microbiol 12:118-123. 10.1111/j.1462-2920.2009.02051.x 
611 Li H. 2013. Aligning sequence reads, clone sequences and assembly contigs with BWA-

612

613

614

615

616

617

618

619

620

621

622

623

624

625

626

627

628

629

630

631

632

633

634

635

636

637

638

639

640

641

642

643

644

645

646

647

648

649

650

651

652

653

654

655

Li H, Handsaker B, Wysoker A, Fennell T, Ruan J, Homer N, Marth G, Abecasis G, Durbin R, and Genome Project Data Processing S. 2009. The Sequence Alignment/Map format and SAMtools. Bioinformatics 25:2078-2079. 10.1093/bioinformatics/btp352

Liesack W, Weyland H, and Stackebrandt E. 1991. Potential risks of gene amplification by PCR as determined by $16 \mathrm{~S}$ rDNA analysis of a mixed-culture of strict barophilic bacteria. Microb Ecol 21:191-198. 10.1007/BF02539153

Lundberg DS, Yourstone S, Mieczkowski P, Jones CD, and Dangl JL. 2013. Practical innovations for high-throughput amplicon sequencing. Nat Methods 10:999-1002. 10.1038/nmeth.2634

Luo C, Knight R, Siljander H, Knip M, Xavier RJ, and Gevers D. 2015. ConStrains identifies microbial strains in metagenomic datasets. Nat Biotechnol 33:10451052. $10.1038 /$ nbt.3319

Magoc T, and Salzberg SL. 2011. FLASH: fast length adjustment of short reads to improve genome assemblies. Bioinformatics 27:2957-2963. 10.1093/bioinformatics/btr507

McDonald D, Price MN, Goodrich J, Nawrocki EP, DeSantis TZ, Probst A, Andersen GL, Knight R, and Hugenholtz P. 2012. An improved Greengenes taxonomy with explicit ranks for ecological and evolutionary analyses of bacteria and archaea. ISME J 6:610-618. 10.1038/ismej.2011.139

Mclnerney P, Adams P, and Hadi MZ. 2014. Error Rate Comparison during Polymerase Chain Reaction by DNA Polymerase. Mol Biol Int 2014:287430. $10.1155 / 2014 / 287430$

Moore LR, Rocap G, and Chisholm SW. 1998. Physiology and molecular phylogeny of coexisting Prochlorococcus ecotypes. Nature 393:464-467. 10.1038/30965

Polz MF, and Cavanaugh CM. 1998. Bias in template-to-product ratios in multitemplate PCR. Appl Environ Microbiol 64:3724-3730.

Schloss PD. 2010. The effects of alignment quality, distance calculation method, sequence filtering, and region on the analysis of 16S rRNA gene-based studies. PLoS Comput Biol 6:e1000844. 10.1371/journal.pcbi.1000844

Schloss PD, Gevers D, and Westcott SL. 2011. Reducing the effects of PCR amplification and sequencing artifacts on 16S rRNA-based studies. PLoS One 6:e27310. 10.1371/journal.pone.0027310

Schloss PD, Jenior ML, Koumpouras CC, Westcott SL, and Highlander SK. 2016. Sequencing $16 \mathrm{~S}$ rRNA gene fragments using the PacBio SMRT DNA sequencing system. PeerJ 4:e1869. 10.7717/peerj.1869

Singer E, Bushnell B, Coleman-Derr D, Bowman B, Bowers RM, Levy A, Gies EA, Cheng JF, Copeland A, Klenk HP, Hallam SJ, Hugenholtz P, Tringe SG, and Woyke T. 2016. High-resolution phylogenetic microbial community profiling. ISME J. 10.1038/ismej.2015.249

Smyth RP, Schlub TE, Grimm A, Venturi V, Chopra A, Mallal S, Davenport MP, and Mak J. 2010. Reducing chimera formation during PCR amplification to ensure accurate genotyping. Gene 469:45-51. 10.1016/j.gene.2010.08.009 
656 Tikhonov M, Leach RW, and Wingreen NS. 2015. Interpreting $16 S$ metagenomic data 657 without clustering to achieve sub-OTU resolution. ISME J 9:68-80. $658 \quad 10.1038 /$ ismej.2014.117

659 Turner S, Pryer KM, Miao VP, and Palmer JD. 1999. Investigating deep phylogenetic 660 relationships among cyanobacteria and plastids by small subunit rRNA sequence 661 analysis. J Eukaryot Microbiol 46:327-338.

662 Weisburg WG, Barns SM, Pelletier DA, and Lane DJ. 1991. 16S ribosomal DNA 663 amplification for phylogenetic study. J Bacteriol 173:697-703.

664 Werner JJ, Koren O, Hugenholtz P, DeSantis TZ, Walters WA, Caporaso JG, Angenent 665 LT, Knight R, and Ley RE. 2012. Impact of training sets on classification of high666 throughput bacterial 16s rRNA gene surveys. ISME $J$ 6:94-103. 10.1038/ismej.2011.82

669 


\section{Figure 1 (on next page)}

Overview of the Long-16S method

A) 16S rRNA gene template molecules are tagged with unique tags via two single rounds of annealing and extension with tagged forward and reverse primers containing random tags (see Figure 2), that also contain Illumina adapter sequences. After removal of tagged primers, tagged templates are amplified via PCR using primers complementary to the adapter sequences. Libraries from one or more samples can then be pooled and sequenced on the MiSeq. Blue triangles and yellow stars indicate random tags of $10 \mathrm{nt}$. B) Full-length 16S rRNA gene amplicon Illumina libraries are tagmented using the standard Nextera method, and two pools of products are amplified which contain either the left end of the tagged amplicons and an internal region, or the right end of the amplicon and an internal region. This procedure adds Nextera adapters for sequencing at the internal end of the fragments. C) Both full-length and tagmented libraries are paired end sequenced, and the unique molecular tags are used to computationally group sequences from the same progenitor 16S rRNA gene molecule for assembly of near full-length sequences. 
Figure 2 (on next page)

Schematic of primers used for molecular tagging of $16 \mathrm{~S}$ rRNA gene template molecules
A) long_forward and B) long_reverse. Stub regions correspond to Illumina adaptors for clustering on the MiSeq, and 0-3 nt phasers are included to increase nucleotide diversity between barcoded samples at individual sequencing cycles. 25 different barcodes were designed using software described in (Meyer \& Kircher 2010) for up to 625 different sample barcode combinations, which are listed in Table S1. 
A

ACACTCTTTCCCTACACGACGCTCTTCCGATCTNNNNNNNNNNGTTGGCCGNNNCGAGAGTTTGATCMTGGCTCAG $2 \mathrm{bp}$

linker

B

p5 stub region

10 bp random $\begin{gathered}8 \mathrm{bp} \\ \text { sample } \\ \text { barcode }\end{gathered}$

$0-3 \mathrm{bp}$ phaser

16S rRNA geneannealing (1391R) CTCGGCATTCCTGCTGAACCGCTCTTCCGATCTNNNNNNNNNNGTTGGCCGNNNTAGACGGGCGGTGTGTRCA 


\section{Figure 3 (on next page)}

Schematic demonstrating the processing of read pairs from the MiSeq to reconstruct near full-length 16S rRNA gene sequences

Read pairs are placed into groups of end+end sequences, or end+internal sequences.

End+end sequences are clustered into groups containing the same combination of random molecular tags from the two ends and putative recombinant clusters are removed (identified as having one or both molecular tags from a separate, more abundant cluster). End+internal sequences are assigned to clusters based on their unique molecular tags, and each cluster is used to generate an assembly of the full-length sequence. 


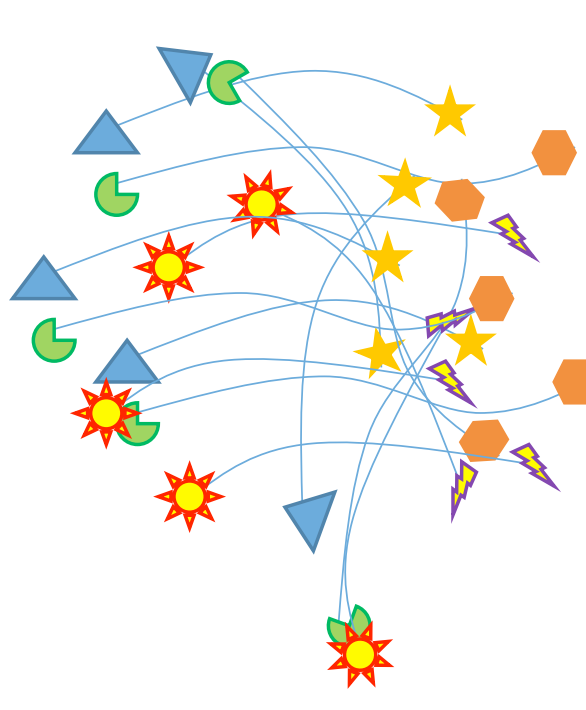

Read pairs from MiSeq nano V2 run $(n=832,293)$

\section{PeerJ}

5

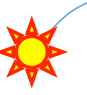

end+end reads $(n=42,715)$

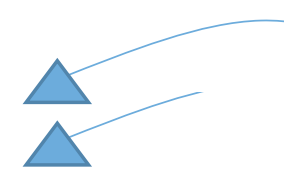

LEnd+Int reads ( $n=444,397)$

REnd+Int reads ( $n=370,297$ )
LEnd+Int barcode clusters $(n=4,361)$

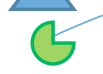

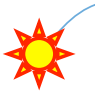

end+end barcode clusters $(n=3,621)$
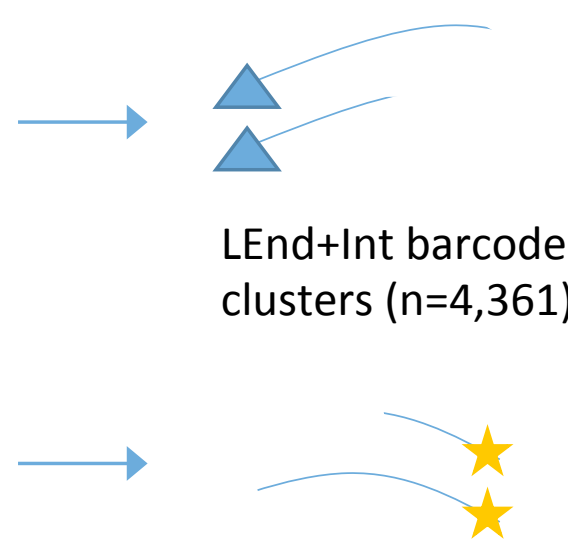

REnd+Int barcode clusters $(n=11,617)$
5

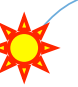

Non-recombinant

(NR) clusters $(n=2,532)$<smiles></smiles>

LEnd+Int read pairs matching NR cluster ( $n=310,246)$

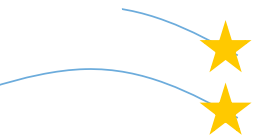

REnd+Int read pairs matching NR cluster $(n=221,859)$
Reconstructed templates $(n=2,304)$

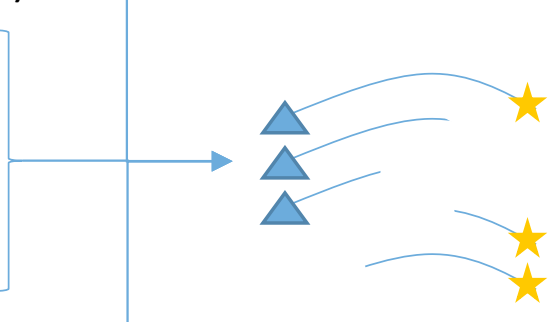


Figure 4 (on next page)

\section{Abundance of putative recombinants}

Violin plot showing the abundance of molecular tag clusters identified as putatively recombinant (left), along with abundances of the progenitor molecules producing recombinant forms. Parental templates were on average 29 times more abundant than the putatively recombinant forms. Median values are indicated by white dotes and the interquartile range by black boxes. 


\section{In-vitro recombination in full length templates}

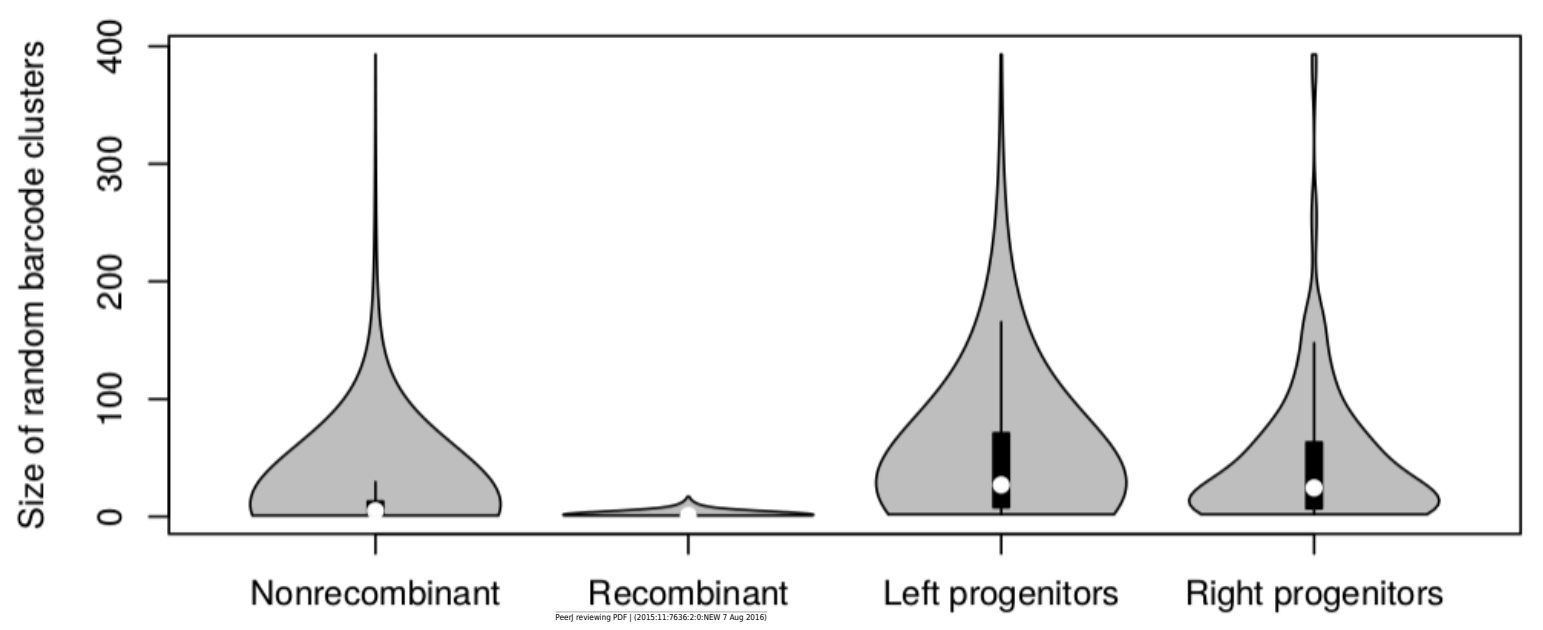




\section{Figure $\mathbf{5}$ (on next page)}

The length distribution of assembled Long-16S sequences

Sequence length ranged from 400 to $1378 \mathrm{nt}$, corresponding to a full-length amplicon. $70 \%$ of the assembled sequences are $>1300 \mathrm{nt}$ in length. 
Figure 6 (on next page)

Quality and coverage plots

A) Quality and B) coverage plots showing median PHRED quality scores and associated coverage across the length of the Long-16S sequences. Higher qualities for the Long-16S sequences are associated with higher coverage, which is particularly apparent at each end of the amplicons (up to 200 and beyond $1200 \mathrm{nt}$ ), which were associated with one read from every read pair in the data set. 


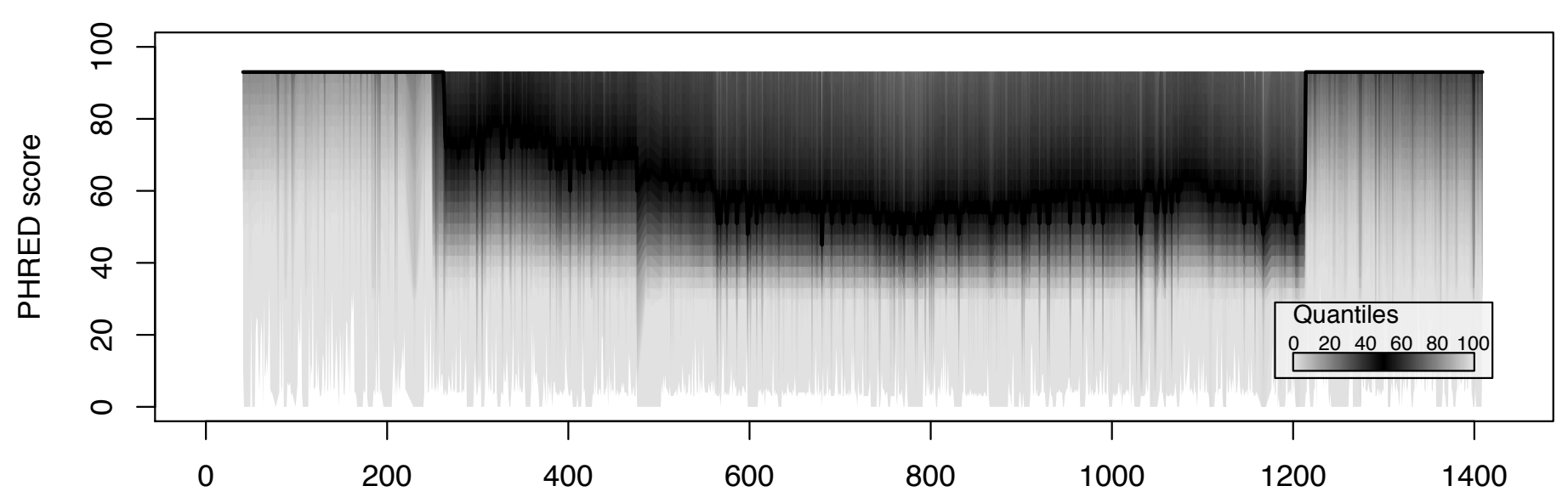

B

Position in Infernal bacterial $16 \mathrm{~S}$ model

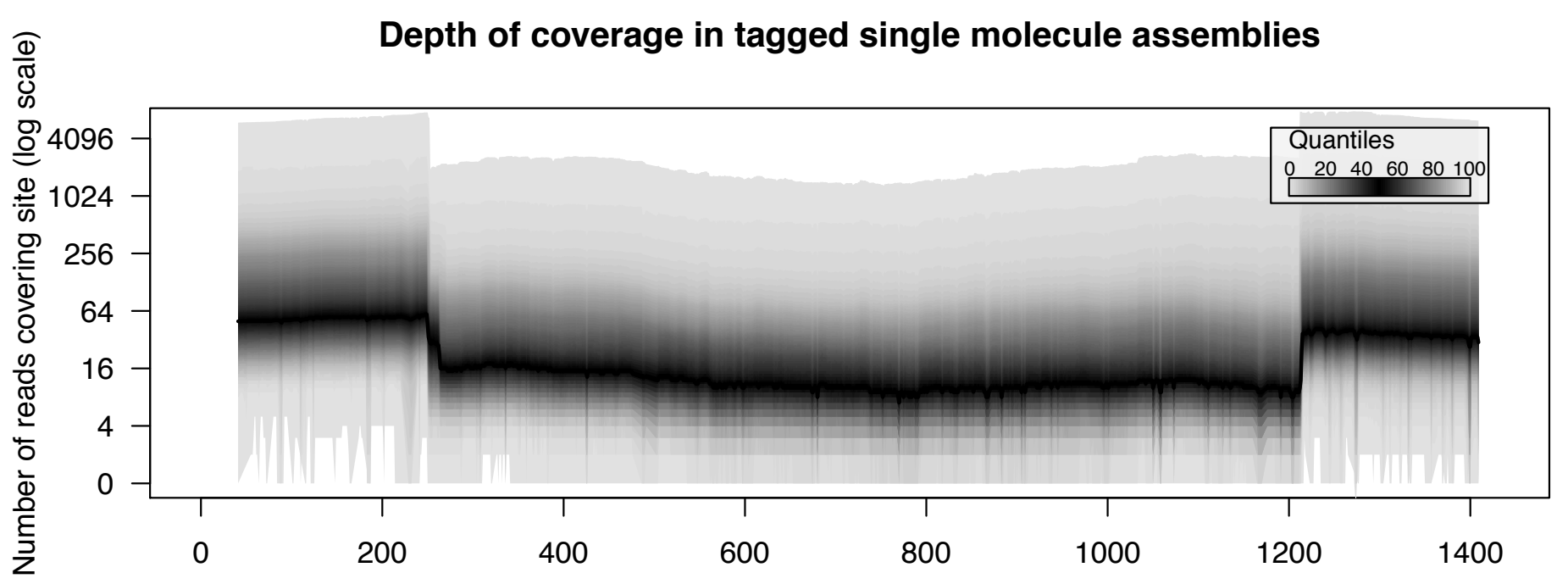


Figure 7 (on next page)

Taxonomic assignments at the phylum and genus level

The relative proportions of taxonomic assignments for (A) phyla and (B) genera are shown for OTUs from V4, Long-16S and extracted-V4 sequences (V4 region extracted from the Long$16 \mathrm{~S}$ sequences). Similar taxonomic assignments are obtained across the $\mathrm{V} 4$ and Long-16S datasets. Only taxa comprising an average of more than $1 \%$ of sequences per sample are displayed. Bars represent the average value across 12 samples for each sequencing method, and error bars are the standard deviation. 
A

Phyla taxonomic assignment

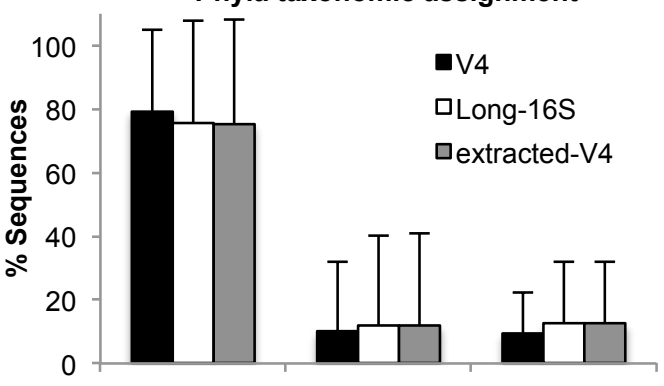

Firmicutes proteobacteria Actinobacteria
B

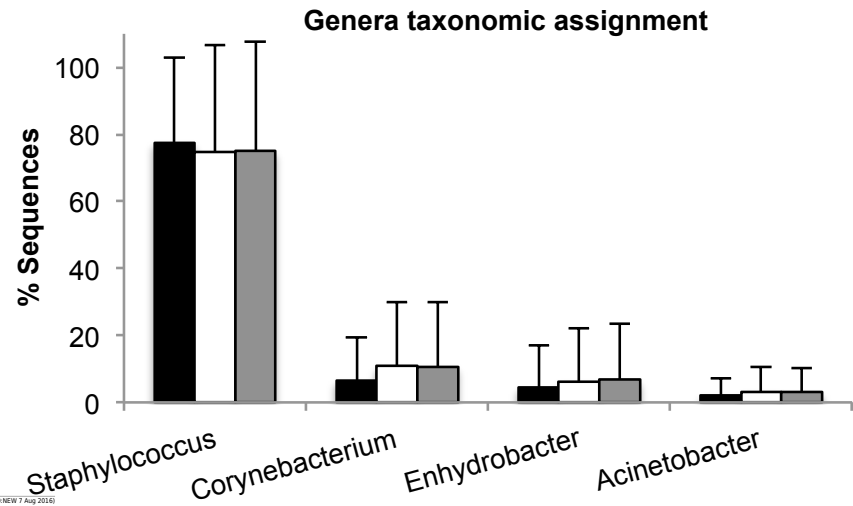




\section{Figure 8 (on next page)}

Distribution of the estimated degree of PCR amplification bias

Estimates of bias were calculated from the deviation of each end+end sequence cluster from the mean end + end sequence cluster abundance. 


\section{PCR amplification bias}

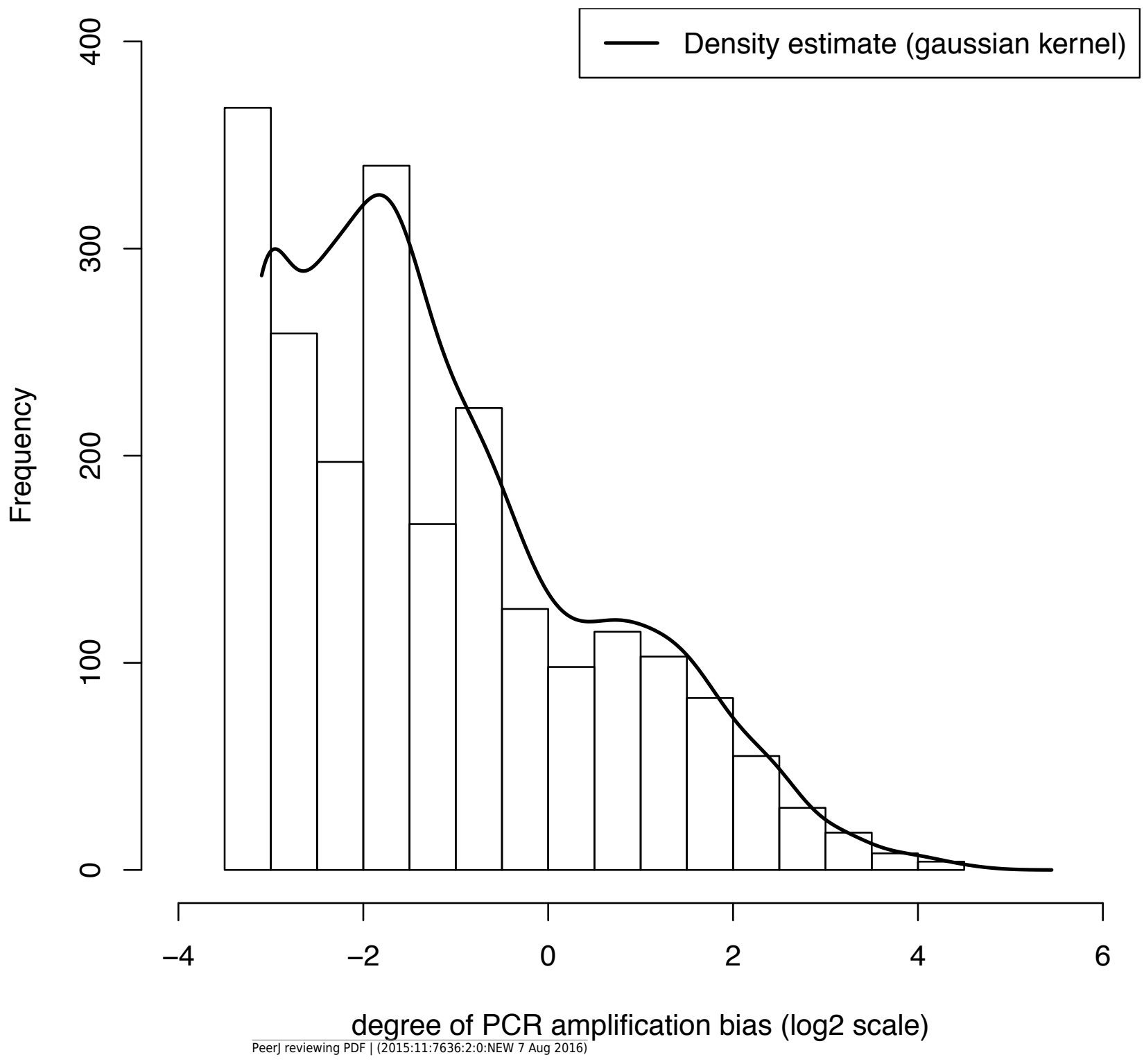

